\title{
The unconscious nature of insight: A dual-task paradigm investigation
}

\author{
Anton A. Lebed ${ }^{\mathrm{a}, \mathrm{b} *}$, Sergei Y. Korovkin ${ }^{\mathrm{a}, \mathrm{c}}$ \\ ${ }^{a}$ Yaroslavl State University, Yaroslavl, Russia \\ ${ }^{\mathrm{b}}$ University of Delaware, Newark, DE, USA \\ ${ }^{c}$ Russian Presidential Academy of National Economy and Public Administration, Moscow, Russia \\ *Corresponding author. E-mail: coglebed@gmail.com
}

Background. Insight is a specific part of the thinking process during creative problem solving. The experience of a sudden unexpected solution of the problem makes it distinct from other problem solving. Though the insight problem solving process is hidden from the observer and the solver himself, it is possible to study working memory changes during the problem-solving process in order to observe the tracks of insight.

Objective. A critical experiment was carried out to determine whether it is legitimate to measure insight-problem-solving dynamics within a dual-task paradigm and working memory model. Also a verification was conducted of the hypothesis of whether insight problem solving competes for cognitive resources with unconscious processes.

Design. We designed a special procedure based on Kahneman's (1973) modified dual-task paradigm, allowing simultaneous performance of the problem-solving process and probe tasks of different types. The reaction time was measured for the probe task. There were two problems conditions (insight and regular), and two probe tasks conditions (implicit and explicit). Participants: 32 participants, aged from 18 to 32 years $(\mathrm{M}=19.81 ; \sigma=2.51)$.

Results. Significant differences in implicit probe reaction time were found between the dual-task condition (implicit categorization and insight problem solving) and solo implicit probe condition $(t(15)=-3.21, p=.006, d=-.76)$. A joint effect of problem type and probe type was found $\left(F(1,60)=4.85, p=.035, \eta_{\mathrm{p}}{ }^{2}=.07\right)$.

Conclusion. The results support the idea that information processing of conscious and of unconscious processes are separate. Unconscious processing capacity is limited. Implicit skill seems to be operated by the same mechanisms as insight problem solving, therefore competing for a common resource. It was also shown that such hidden creative unconscious processes as insight can be tracked via working memory load.

Keywords: insight, thinking, dual-task, implicit learning, working memory, problem solving 


\section{Introduction}

Insight has always been one of the most mysterious phenomena in the psychology of thinking. Its mechanisms, role, and structure are still uncertain. Some investigators consider that there is no special problem type known as "insight". The cybernetic model of Newell and Simon (1972) attempted to describe the role and processing of insightful solutions in the terms of regular problem solving. They suggested that there is no specific method of insight problem solving, so it can be explained in terms including operators, heuristics, and problem space. However, many critics of this view have noticed that creative problem solving is often indescribable in the common terms of a cybernetic model, as it lacks visible structure.

Many other investigators have approached insight as a special specific phenomenon in a separate class of creative problems known as insight problem solving. Researchers vary a lot, for example using different stimuli, but methods that are completely alike. Chu and MacGregor (2012) present an overview of the bestknown methods and problems used to investigate insight. They made a catalog of problems, varying in difficulty and representation type: classical problems such as the six matchstick problem, verbal riddles, and spatial puzzles. These problems vary greatly, making it hard to integrate data from several experiments into one conclusion. The new problems include matchstick arithmetic and compound remote associations. The authors compare theories of insight, concluding that there is no complete answer yet to the problem of insight.

Since the identification of insight as a subject for psychological study, there have been few investigations of its mechanisms and structure. The main reason for this lies in its unconscious nature, making it very difficult to gather data about it. Even the problem-solver cannot realize how far he is from the solution, as shown in the "feeling of warmth" investigation by Metcalfe and Wiebe (1987). They showed that participants were unable to adequately measure how close they are to the answer in insight problem solving, even though they gave rather precise estimates during regular problem solving.

One of the key questions in understanding the nature of insight consists in figuring out what processes precede insight, what makes it more difficult, and what allows a person to overcome an impasse in the problem-solving process. Empirically, insight problem solving is described in terms of multiple impasses that the solver encounters after using all available options, followed by incubation, which is characterized by very limited conscious thinking. There are various points of view on the nature of incubation and concurring processes.

On the one hand, there are doubts about the very existence of some specific active processes in incubation. According to this point of view, incubation itself consists of nothing special, nothing more than forgetting incorrect solution attempts and options (Anderson, 2010; Simon, 1977; Woodworth, 1938). This approach assumes that incubation is a temporary period, during which heuristics, solving strategies, and after-effects of incorrect solutions are erased. Mental fixation, occurring in early stages of problem solving, can be overcome by just forgetting actual schemas; therefore, the effectiveness of problem solving is directly related to the duration of incubation (Dodds, Ward, \& Smith, 2004; Sio \& Ormerod, 2009). Some believe that incubation is required for an attention switch, rather than forgetting 
(Segal, 2004). The attention switch is supposed to be relatively fast and spontaneous. This idea predicts the absence of a positive relation between problem-solving effectiveness and incubation duration. In memory erase models, working memory is considered to play only a minor role. There are other possible explanations for the lack of visible activities during incubation within the "nothing special" approach: unsolved problems might be stored in memory in order to look for problem-relevant environmental cues that would make it possible to solve the problem using new information (Seifert, Meyer, Davidson, Patalano, \& Yaniv, 1995). According to this point of view, incubation is considered an active process of awaiting relevant information that requires some usage of long-term working memory.

On the other hand, there are models of insight problem solving incubation that assume specific unconscious processes that are distinct from conscious mechanisms. There are multiple data suggesting that a solution can be found before the solver is aware of it. Thus, anagram investigation with eye-tracking showed that participants tend to focus their attention on the solution part of the anagram some time prior to the actual solution (Ellis, 2012; Ellis, Glaholt, \& Reingold, 2011). These authors conclude that there is unconscious knowledge of the solution prior to the solution itself. Additionally, there is evidence that unconscious hints can greatly affect insight problem solving (Bowden, 1997; Thomas \& Lleras, 2009; Werner \& Raab, 2013), while verbal hints are ineffective (Weisberg \& Alba, 1981). The time at which the hint is presented can also affect its effectiveness. The solver has to spend some time working on the problem to be able to understand the hint, while spending too much time increases the effect of incorrect solutions (Moss, Kotovsky, \& Cagan, 2011). When solving arithmetical problems, children are capable of using new methods and solving strategies long before they become aware of this ability and are able to report it (Siegler, 2000).

Even considering substantial evidence supporting the idea of unconscious processes of insight incubation, there is no common model of unconscious information processing in problem solving. One approach is based on the idea of two separate parallel, yet competing, systems: System 1 and System 2 (Kahneman, 2011) or conscious and unconscious modes of thought (Dijksterhuis \& Nordgren, 2006). According to these models, drawing attention from or overloading conscious thought can facilitate unconscious information processing. At the same time, conscious (logical) and unconscious (intuitive) thinking can be considered not only as independent systems, but also as levels/layers of one process (Ponomarev, 1976). During the creative problem-solving process, the solver "climbs" from infantile forms of intellect towards more ontologically mature methods. In case of failure to solve a problem, the solver returns to the lowest levels of thinking. Another approach attempts to describe the processes underlying incubation in terms of semantic networks and neural networks (Hélie \& Sun, 2010; Martindale, 1995; Sio \& Rudowicz, 2007). The process of incubation is considered a gradual or rapid change of activation in the network and the creation of distant associations. This approach explains the effect of semantic hints during problem incubation, while other effects are much less readily interpretable in these terms. The mechanisms of unconscious processing are usually described in general terms and are hard to prove experimentally. Some of the known mechanisms of insight solution, such as constraint relaxation (disabling rules, supported by functional fixedness) and chunk 
decomposition (dividing up pieces of information that are perceived as a whole) (Knoblich, Ohlsson, \& Raney, 2001; Öllinger, Jones, Faber, \& Knoblich, 2013) are hard to consider either exclusively conscious or unconscious. One of the potential reasons why the data acquired by different researchers are so controversial is the use of different stimulus materials (problems of different sorts), different incubation models, and different approaches to affect incubation. Additionally, different stages of problem solving might have different underlying processes. These issues lead to the question, whether it is possible to investigate thinking processes using one single problem scenario.

Korovkin, Vladimirov, and Savinova (2014) investigated the insight-problemsolving process within a dual-task paradigm, showing the differences between insight problem solving and regular problem solving. The participants were asked to solve either an insight or regular (algorithmized) problem and to perform a probe task at the same time. The solution time was divided into 10 stages, each representing the average reaction time for a probe task at different moments of the solution. They found that there is a significant difference between the last stages of insight problem solving and regular problem solving.

Other experiments investigated different mental storage systems by varying the probe task material and the problem types. Some experiments were designed to discover whether the information contained in working memory blocks can be put into a state of competition by attaching probe tasks of various kinds.

However, some have criticized the procedures of such investigations. According to cognitive unconscious theory (Allakhverdov, 2009), there are two big obstacles to measuring insight within a dual-task paradigm. First of all, unconscious processes are considered limitless by cognitive unconscious theory, making it impossible to create any competition between insight problem solving and the probe task. Secondly, the author pointed to the conscious nature of working memory, concluding that it is not legitimate to investigate an unconscious process - insight - by having it compete with working memory probe tasks.

Indeed, the original working memory models considered this memory as fully conscious. Working memory was introduced by Baddeley and Hitch (1974) as a system providing temporary storage for manipulations of information required for complex cognitive problem solving. It consists of central executive control and three subsystems: a phonological loop that processes verbal data; a visuospatial sketchpad that processes visual data and spatial relations; and an episodic buffer that combines all the types of information to create a working space. Later on, several attempts were made to investigate the role of working memory in unconscious thinking. Some authors used an implicit learning paradigm to study this. Being an unconscious process, implicit learning might give investigators a clue about the role of working memory load in the learning process (Reber, 1967). It was discovered that working memory overload negatively affects implicit learning (Reber, \& Kotovsky, 1997) and that implicit skill might be stored in working memory (Hassin, Bargh, Engell, \& McCulloch, 2009).

In our studies, we use Kahneman's (1973) resource competition model as well as a probe task method. The resource model supposes that there is a limited cognitive resource, used by most mental processes that compete for it. According to its author, this resource has a biological basis: arousal. In a situation of resource defi- 
cit, task evaluation occurs, so the most relevant tasks receive the most resources. He demonstrated his model with a simple experiment using a dual-task paradigm: simultaneous processing of mental problem solving and a simple probe task. It turned out that the effectiveness of solving the probe task dramatically decreases in the middle of the primary problem solving, because this period requires the greatest amount of resources. Based on the amount of mistakes (in the probe task) in this period, he concluded that all available resources were allocated to the primary problem, leaving the probe task unsupported.

\section{Method}

According to the above-mentioned investigations, we decided that implicit learning might be a fitting probe task to interact with the insight-problem-solving process through the means of working memory load. The experimental procedure combines two paradigms: the working memory competition paradigm and the dual-task paradigm, allowing us to investigate the thinking process during the problem-solving process based on reaction time and mistakes in the probe task performed at the same time as the participant solves the problem.

There are several requirements for any probe task. It should be:

1) Rapid. The more often the participant is required to react for the probe task, the more descriptive dynamics we can receive.

2) Congruent. The probe task must be relevant to the experimental problem in order to achieve some interaction with it.

3) Simple. The probe task should not distract from the problem-solving process; otherwise, the whole solution will be uninformative, because of its unnatural processing.

Our main goal was to find out whether there is competition between unconscious processes and insight problem solving. We have chosen implicit skill to represent unconscious processes.

According to the dual-task paradigm, the participants had to perform the problem solving and the probe task at the same time. As the participant reads and thinks about the primary problem, the probe task appears on the screen, requiring constant reactions for changing stimuli. The probe task is usually a simple decision or categorization task that requires minimum thinking and limited input variations of two or three buttons. Based on the reaction time of the probe task, we can judge participants' mental tension (representing working memory load) at the different stages of the solution process. The greater the reaction time for the probe task, the less free resources are available for it because of the increased resource-requirements of the primary task. This method allows us to observe the dynamics of the thinking process in detail, but in this paper, we discuss the efficiency of the probe task performance without respect to its dynamics.

The study sample consisted of 32 participants, aged from 18 to 32 years $(M=19.81 ; \sigma=2.51)$, with 7 men and 25 women: students, graduate students, and other people with higher education. Participants received no additional motivation. They were initially seated approximately $45 \mathrm{~cm}$ from the monitor, but were free to 
move their heads during the experiment; therefore, the visual angle subtended by the probe task was not controlled. Stimulus size varied from $5 \mathrm{~cm}$ to $9 \mathrm{~cm}$ depending on the categorization features.

To test the validity of the dual-task paradigm application in insight problem solving, we hypothesized that the probe task can compete with insight problem solving for common resources. Based on the idea of two relatively separate systems of information processing in the human mind, we also hypothesized that insight problem solving uses specific unconscious/implicit working memory resources.

To verify this hypothesis, we created a computer program in the Python environment with PsychoPy application v1.76.00 (Peirce, 2007). We varied the working memory load type with the probe task: either implicit with unconscious selection criteria or explicit with conscious selection criteria. We also varied the problem type: either insight or regular problems. The problems can be found in Appendix A. We measured probe task reaction time and average solution time. There were four experimental groups: implicit probe task with insight problem, explicit probe task with regular problem, implicit probe task with regular problem, and explicit probe task with insight problem.

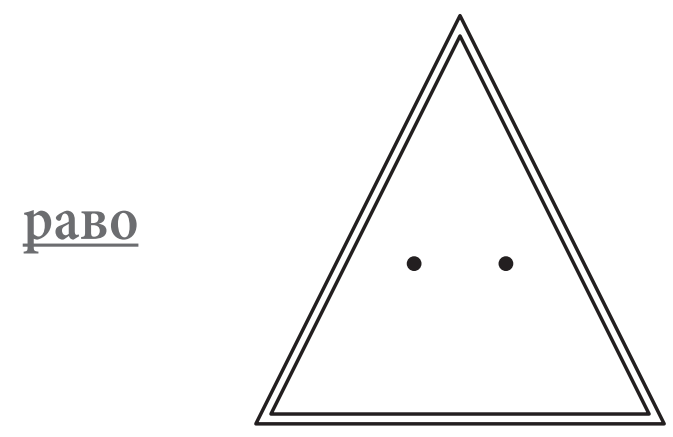

Figure 1. Probe tasks examples (verbal probe task and figures probe task)

The probe task examples are illustrated in Figure 1. There were two kinds of probe tasks, to avoid stimulus-specific results: figures and nonsense syllables. The participants were asked to group both figures and nonsense syllables into either "left" or "right" categories, based on a certain rule; the probe stimulus could vary in color, form, size, and additional markers. Only a certain combination of these properties was considered right, the rest were considered left. The categorization criteria are stated in Appendix B.

The rules were shown to the participants in the "explicit" groups and were hidden from those in the "implicit" groups. During a practice trial, the implicit group performed an implicit learning sequence. The participants were asked to categorize the stimuli and had audial feedback: a ring-sound if the answer was correct and a drum-sound if the answer was wrong. The sequence was considered complete when the participants were able to produce correct answers in no less then 59\% of the trials. Participants passed two trials in our experiment: the first one was a practice (control) trial and the second one was experimental. During the practice trial, the participants had to become familiarized with the experimental stimuli or 
to develop an implicit skill (in the implicit probe task group). Once the participants were familiar with the probe task, the second trial began: simultaneous performing the probe task and the primary problem. The participants had to push buttons to categorize the stimuli that popped up and to solve the primary problem.

There were either creative insight problems, or regular problems that required simple mathematical calculation. Regular problems feature distinctive sequential steps that are required to solve them. These steps are known to the solver, along with all possible actions within the problem space. The procedure/sequence for the solution can be described in terms of algorithms. Insight problems, on the contrary, require a substantial change of problem representation, possible actions or their applications (a functional solution is required), while the final goal, representation, or possible actions are not initially obvious to the solver. An example of an insight problem: "Misha and Sasha played in the basement. It was dark and dirty in there. Once they got upstairs, Sasha's face turned out to be very dirty, but Misha's face was clean. Nevertheless, only Misha went to wash his face, but not Sasha. Why?" An example of a regular problem: "Three chickens lay three eggs in three days. How many eggs will 12 chickens lay in 12 days?" There were two problems of each type, to avoid problem-specific results. All the problems had been chosen as having the same success rate and solution time. The problems were presented in the form of a text on the screen.

\section{Results}

Statistical analysis was performed using two-way ANOVA and $t$-test for dependent and independent samples. We received significant results, providing evidence on the researched goal: there is a significant difference or probe task reaction time between the control condition of implicit practice and the experimental condition of simultaneous performance of implicit probe task with insight problem $(t(15)=-3.21, p=.006, d=-.76)$. Average reaction time for a probe task is significantly higher in dual-task conditions compared to control single-task conditions (see Table 1). This means that insight problem solving and the implicit probe task compete for the cognitive resources of working memory. But surprisingly, we no found differences between practice conditions and the experimental condition of simultaneous performance of implicit $(t(15)=-.27$,

Table 1. Average reaction time of probe task performance

\begin{tabular}{|c|c|c|c|c|}
\hline & \multicolumn{2}{|c|}{ Implicit probe task } & \multicolumn{2}{|c|}{ Explicit probe task } \\
\hline & Insight problem & Regular problem & Insight problem & Regular problem \\
\hline Conditions & $\mathrm{M}(\mathrm{SD})$ & $\mathrm{M}(\mathrm{SD})$ & $\mathrm{M}(\mathrm{SD})$ & $\mathrm{M}(\mathrm{SD})$ \\
\hline $\begin{array}{l}\text { Practice (without } \\
\text { problem solving) }\end{array}$ & $1.07(0.44)$ & $1.24(0.49)$ & $2.64(0.95)$ & $2.52(0.95)$ \\
\hline $\begin{array}{l}\text { Experiment(with } \\
\text { problem solving) }\end{array}$ & $1.55(0.78)$ & $1.3(0.79)$ & $2.37(1.11)$ & $1.99(0.88)$ \\
\hline
\end{tabular}

Note. $\mathrm{M}$ - mean, $\mathrm{SD}$ - standard deviation 
$p=.79, d=-.06)$ and explicit $(t(15)=.93, p=.37, d=.26)$ probe tasks with regular problems. Counterintuitive results were found in comparison between explicit practice and the experimental condition of simultaneous performance of an explicit probe task with an insight problem $(t(15)=2.94, p=.01, d=.58)$. In the case of an explicit probe task in insight problem solving, we found that the reaction time in the dual-task condition is significantly less. We propose that the probes were too difficult to perform them simultaneously with primary problem solving. Thus, our participants might have stopped paying attention to the difficult secondary probe task altogether.

We further examined how problem type and probe task type affect the average solution time using ANOVA (see Table 2). We found that probe task type significantly affects the average solution time $\left(F(1,60)=4.85, p=.035, \eta_{\mathrm{p}}{ }^{2}=.07\right)^{1}$. At the same time, the problem type effect is not significant $(F(1,60)=.16, p=.69$, $\left.\eta_{\mathrm{p}}{ }^{2}=.002\right)$ and there is no joint effect of both probe task type and problem type $\left(F(1,60)=2.83, p=.098, \eta_{\mathrm{p}}{ }^{2}=.05\right)$.

Table 2. Average solution time of regular and insight problems

\begin{tabular}{ccc}
\hline & Insight problem & Regular problem \\
\cline { 2 - 3 } Probe task & M (SD) & M (SD) \\
\hline Implicit & $354.14(276.94)$ & $169.19(178.16)$ \\
Explicit & $400.28(242.52)$ & $513.92(580.52)$ \\
\hline
\end{tabular}

Note. $\mathrm{M}$ - mean, $\mathrm{SD}$ - standard deviation

Pairwise comparison using $t$-test shows significant differences in the average solution time between regular problem solving during implicit probe task performance and other conditions: regular problem with explicit probe task $(t(30)=-2.27$, $p=.03, d=-.8)$, insight problem with explicit probe task $(t(30)=-3.07, p=.005$, $d=-1.09)$, and insight problem with implicit probe task $(t(30)=-2.25, p=.03$, $d=-.79)$. No other significant differences between groups were found.

\section{Conclusion}

As we can see from the results by comparing the practice and dual-task conditions, implicit learning probe performance was significantly impaired by the presence of insight problem solving. This kind of interaction of similar information type can be explained by resource competition in working memory. Though the original working memory is considered to have only three blocks for situational information - a phonological loop, a visuospatial sketchpad, and an episodic buffer they do not account for consciousness of this information. As there is no division into conscious and unconscious information types in working memory model, it cannot fully explain the competition we found in our experiment. However there are extensions to the classical working memory model: Global Workspace theory

$1 \quad \eta_{\mathrm{p}}{ }^{2}-$ Partial eta-squared (effect size). 
and Intelligent Distributive Agent theory (Baars, \& Franklin, 2003). Global Workspace theory suggests that there is a special workspace where conscious perception, imagery, inner speech, and reportable goals are processed and kept. Other working memory blocks are used in "consciousness cycles", sending unconscious information into the working space. In terms of an updated working memory model, an interaction of conscious and unconscious processing is possible by means of competition for resource (Intelligent Distributive Agent), which is supported by our empirical data. Another working memory model that accounts for implicit processes features the Implicit Working Memory construct (Hassin et al. 2009). Implicit Working Memory was shown to be involved in performing tasks outside of awareness. It seems that insight problem solving involves a great deal of implicit processing (as shown by competition with implicit learning), leading to the conclusion that implicit working memory tasks can be successfully used as a probe in a dual-task paradigm studying insight problem solving.

On the contrary, there was no difference between explicit probe performance in training conditions and in dual-task conditions. This might have two reasons: assuming that decision making using explicit rules is primarily a System 2 activity, it features, firstly, fast learning (which allows compensation for dual-task conditions by rapid increase in skill), and, secondly, conscious control availability (which allows participants to maintain the same performance by decreasing precision in the probe task). Both suggestions, however, require further empirical verification.

Other results suggest that participants had substantial difficulties with insight problem solving while performing implicit categorization, and with regular problem solving while performing explicit categorization. Regular and insight problem solving seem to have different involvement in System 1 and System 2. Because these two systems (referred to as Default Network and Control Network) were shown to be very distinctive in terms of activation (Gu et al., 2015), two dual-task activities that are processed within the same system (e.g., insight problem solving and implicit learning) have to share and compete for activation, whereas performing activities processed by different systems (e.g., implicit learning and regular problem solving) involves much less interference. In other words, conscious and unconscious information processing have distinct capacities and underlying neurological bases. Conceptually, regular problem solving might rely more on such conscious features, as attention or working memory, while insight problem solving requires implicit and/or bottom-up processing. This suggestion is consistent with the common view of the unconscious and sudden nature of insight.

Further experiments should aim at a more careful and precise load of working memory blocks in order to obtain data about insight solution requirements in working memory.

\section{Limitations}

The limitations of our study are common for working memory model investigations and insight problem solving. Working memory is a complex system that is hard to affect precisely, loading one of its blocks and avoiding others. Insight problem solving is often accompanied by discussions of where the problem stimuli 
could really be called insightful, as there are no complete descriptions and common views on insight problems. The issue of the problem stimuli can be solved either by using only problems that are commonly considered insightful or by introducing a list of criteria for such problems. The other issue of insight problem is the lack of a "eureka" experience in some participants, although they successfully solved the insight problem.

\section{Future directions}

There are multiple possible prospective directions of this study; first, having an implicit dual-task probe as a tool to interact with unconscious information processing, the dynamic features of the insight incubation processes can be investigated and discovered. Second, dual-process theories can greatly benefit from this method, as it allows dissociating System 1 (implicit processing) from System 2 (explicit processing) in a broad variety of activities.

The results obtained lead to the conclusion that the probe task method is appropriate for investigation of insight problem solving by means of creating working memory competition between a probe task and problem solving leading to measurable change in reaction time. Therefore, it can be specifically used to investigate the problem of unconscious incubation. The probe task, however, should have a low complexity level to avoid withdrawing too much of the resource (working memory) from the primary task and thereby interfering with the thinking process. Developing such probe tasks will allow us to observe a stable, visible working memory load pattern during the insight incubation process and, therefore, is a goal of our future research. Distinctive features of the probe task should be relevant to the primary task - an insight problem - and, more specifically, to the processes that occur during its incubation.

\section{Acknowledgements}

This work is supported by the Russian Science Foundation (16-18-10030) and the State Assignment (25.5666.2017/BP).

\section{References}

Allakhverdov, V.M. (2009). Razmishleniya o nauke psihologii s vosklicatel'-nim znakom. [Discussions about psychology]. Saint Petersburg: Format.

Anderson, J.R. (2010). Cognitive Psychology and its implications (7th ed.). New York: Worth Publishers.

Baars, B.J., \& Franklin, S. (2003). How conscious experience and working memory interact. Trends in Cognitive Science, 7(4), 166-172. doi: 10.1016/S1364-6613(03)00056-1

Baddeley, A.D., \& Hitch, G.J. (1974).Working memory. In G.H. Bower (Ed.). The Psychology of Learning and Motivation. Vol. 8. NY: Academic Press, 47-89. doi: 10.1016/S0079-7421(08)60452-1

Bowden, E.M. (1997). The effect of reportable and unreportable hints on anagram solution and the Aha! experience. Consciousness and Cognition, 6(6), 545-573. doi:10.1006/ ccog. 1997.0325 
Chu, Y., \& MacGregor, J.N. (2011) Human performance on insight problem solving: A review. The Journal of Problem Solving, 3(2), 119-150. doi: 10.7771/1932-6246.1094

Dijksterhuis, A., \& Nordgren, L.F. (2006). A theory of unconscious thought. Perspectives on Psychological Science, 1(2), 95-109. doi:10.1111/j.1745-6916.2006.00007.x

Dodds, R.A., Ward, T.B., \& Smith, S.M. (2004). A review of the experimental literature of incubation in problem solving and creativity. In M.A. Runco (Ed.), Creativity research handbook. (3rd ed., pp. 251-284). Cresskill, NJ: Hampton.

Ellis, J.J., Glaholt, M.G., \& Reingold, E.M. (2011). Eye movements reveal solution knowledge prior to insight. Consciousness and Cognition, 20(3), 768-776. doi: 10.1016/j.concog.2010.12.007

Gu, S., Pasqualetti, F., Cieslak, M., Telesford, Q.K., Alfred, B.Y., Kahn, A.E., ... \& Bassett, D.S. (2015). Controllability of structural brain networks. Nature Communications, 6. doi:10.1038/ ncomms 9414

Hassin, R.R., Bargh, J.A., Engell, A.D., \& McCulloch, K.C. (2009). Implicit working memory. Consciousness and Cognition, 18(3), 665-678. doi: 10.1016/j.concog.2009.04.003

Hélie, S., \& Sun, R. (2010). Incubation, insight, and creative problem solving: A unified theory and a connectionist model. Psychological Review, 117(3), 994-1024. doi:10.1037/a0019532

Kahneman D. (1973) Attention and effort. Englewood Cliffs, NJ: Prentice Hall.

Kahneman, D. (2011). Thinking, fast and slow. New York: Farrar, Straus and Giroux.

Knoblich, G., Ohlsson, S., Raney, G.E. (2001). An eye movement study of insight problem solving. Memory \& Cognition, 29(7), 1000-1009. doi: 10.3758/BF03195762

Korovkin, S., Vladimirov, I., \& Savinova, A. (2014). The dynamics of working memory load in insight problem solving. The Russian Journal of Cognitive Science, 1(4), 67-81.

Martindale, C. (1995). Creativity and connectionism. In S. M. Smith, T.B. Ward, \& R.A. Finke (Eds.), The Creative Cognition Approach (249-268). Cambridge, MA: MIT Press.

Metcalfe, J., Wiebe, D. (1987). Intuition in insight and noninsight problem solving. Memory \& Cognition, 15(3), 238-246. doi: 10.3758/BF03197722

Moss, J., Kotovsky, K., \& Cagan, J. (2011). The effect of incidental hints when problems are suspended before, during, or after an impasse. Journal of Experimental Psychology: Learning, Memory, and Cognition, 37(1), 140-148. doi:10.1037/a0021206

Newell, A., Simon, H.A. (1972). Human problem solving. Englewood Cliffs, NJ: Prentice-Hall.

Öllinger, M., Jones, G., Faber, A.H., \& Knoblich, G. (2013). Cognitive mechanisms of insight: the role of heuristics and representational change in solving the eight-coin problem. Journal of Experimental Psychology: Learning, Memory, and Cognition, 39(3), 931-939. doi: 10.1037/ a0029194

Peirce, J.W. (2007) PsychoPy - psychophysics software in Python. Journal of Neuroscience Methods, 162(1-2), 8-13. doi: 10.1016/j.jneumeth.2006.11.017

Ponomarev, Y.A. (1976). Psikhologiya tvorchestva [The Psychology of creativity]. Moscow, Nauka.

Reber, A.S. (1967). Implicit learning of artificial grammars. Journal of Verbal Learning and Verbal Behavior, 6(6), 855-863. doi: 10.1016/S0022-5371(67)80149-X

Reber, P.J., \& Kotovsky, K. (1997). Implicit learning in problem solving: The role of working memory capacity. Journal of Experimental. Psychology: General. 126(2). 178-203. doi: 10.1037/0096-3445.126.2.178

Segal, E. (2004). Incubation in insight problem solving. Creativity Research Journal, 16(1), 141148. doi:10.1207/s15326934crj1601_13

Seifert, C.M., Meyer, D.E., Davidson, N., Patalano, A.L., \& Yaniv, I. (1995). Demystification of cognitive insight: opportunistic assimilation and the prepared-mind perspective. In R.J. Sternberg \& J.E. Davidson (Eds.), The Nature of Insight (65-124). Cambridge, MA: MIT Press. 
Siegler, R.S. (2000). Unconscious insights. Current Directions in Psychological Science, 9(3), 7983. doi:10.1111/1467-8721.00065

Simon, H.A. (1977). Scientific discovery and the psychology of problem solving. In Models of Discovery and other topics in the methods of science (pp. 286-303). Springer Netherlands. doi:10.1007/978-94-010-9521-1_16

Sio, U.N., \& Ormerod, T.C. (2009). Does incubation enhance problem solving? A meta-analytic review. Psychological Bulletin, 135(1), 94-120. doi:10.1037/a0014212

Sio, U.N., \& Rudowicz, E. (2007). The role of an incubation period in creative problem solving. Creativity Research Journal, 19(2-3), 307-318. doi:10.1080/10400410701397453

Thomas, L.E., \& Lleras, A. (2009). Covert shifts of attention function as an implicit aid to insight. Cognition, 111(2), 168-174. doi:10.1016/j.cognition.2009.01.005

Weisberg, R.W., \& Alba, J.W. (1981). An examination of the alleged role of "fixation" in the solution of several "insight" problems. Journal of Experimental Psychology: General, 110(2), 169-192. doi:10.1037/0096-3445.110.2.169

Werner, K., \& Raab, M. (2013). Moving to solution: effects of movement priming on problem solving. Experimental Psychology, 60(6), 403-409. doi:10.1027/1618-3169/a000213

Woodworth, R.S. (1938). Experimental psychology. New York: Holt.

Original manuscript received May 15, 2015

Revised manuscript accepted June 06, 2016

First published online September 30, 2017 


\section{Appendix A. Problems}

Regular problems:

1) Calculate: $25^{\star} 65=$ ?

2) Three chickens produce three eggs in three days. How many eggs would 12 chicken produce in 12 days?

Insight problems:

1) Misha and Sasha were playing in the attic. It was dark and dirty in there. When they came downstairs, Sasha's face was covered with dust, while Misha's face was clean. Nevertheless, only Misha decided to wash his face. Why?

2) A magician has put 11 coins on the table. He asks spectators to remove five coins out of 11 and add four coins in such way that nine coins remain. How should they do this?

\section{Appendix B. Rules of categorization in explicit probe task}

For both categorization stimuli, there was a single rule to determine the correct category of the presented object. Each object had four features with three possible options within each feature. Only one of three options was considered correct, except for one feature that had two possible correct options to decrease the learning difficulty. If the presented object was correct according to two or more features, it was considered correct for the categorization task.

Specifically, the features and their correct options (in bold) were the following:

For words stimuli

1) Word length - 3 letter, 4 letters, 5 letters

2) Size - small, medium, huge

3) Font - italic, bold, regular

4) Color - green, black, red

For figures stimuli

1) Shape - circle, square, triangle

2) Border lines - one thin line, two thin lines, one thick line

3) Dots inside - no dots, one dot, two dots

4) Color - black, green, red 